\title{
Bosentan does not improve pulmonary hypertension and lung remodeling in heart failure
}

\author{
B.H. Jiang, J-C. Tardif, Y. Shi and J. Dupuis
}

ABSTRACT: Pulmonary hypertension $(\mathrm{PH})$ and right ventricular $(\mathrm{RV})$ dysfunction associated with heart failure (HF) carry a poor prognosis. Although endothelin receptor antagonists (ERAs) demonstrated benefits in pulmonary arterial hypertension, their efficacy in $\mathrm{PH}$ associated with $\mathrm{HF}$ was not specifically evaluated.

2 weeks after myocardial infarction (MI) rats received bosentan $\left(100\right.$ or $200 \mathrm{mg} \cdot \mathrm{kg}^{-1} \cdot \mathrm{day}^{-1}$ ) or no treatment for 3 weeks. $\mathrm{PH}, \mathrm{RV}$ hypertrophy and function as well as lung remodeling and function were evaluated.

LV echocardiographic wall motion abnormality and function measured before treatment (2 weeks after MI) and after treatment (5 weeks after $\mathrm{MI}$ ) were similar in $\mathrm{MI}$ control and $\mathrm{MI}$ treatment groups. HF induced PH and RV hypertrophy compared with sham: RV systolic pressure $39 \pm 5$ versus $23 \pm 0.8 \mathrm{mmHg}$ and $\mathrm{RV} /$ left ventricular+septum weight $52 \pm 7$ versus $24 \pm 0.5 \%$ (all $\mathbf{p}<\mathbf{0 . 0 1}$ ). Bosentan did not significantly modify these parameters. In addition, bosentan did not improve depressed RV function measured by echocardiograph from the RV myocardial performance index and tricuspid annular plane systolic excursion. The respiratory pressurevolume relationship revealed that HF caused a restrictive lung syndrome with histological lung remodeling and fibrosis, also not improved by bosentan.

Dual ERA therapy with bosentan does not reduce $\mathrm{PH}$, RV hypertrophy and lung remodeling and dysfunction associated with ischaemic HF.

\section{KEYWORDS: Bosentan, endothelin receptor antagonist, pulmonary venous hypertension}

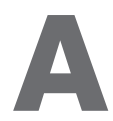

lthough endothelin receptor antagonists (ERAs) demonstrated benefits in pulmonary arterial hypertension $(\mathrm{PAH})$, their efficacy in pulmonary hypertension $(\mathrm{PH})$ associated with heart failure (HF) has not been specifically evaluated. We hypothesised that ERAs may selectively improve $\mathrm{PH}$ and right ventricular (RV) function by reducing lung remodeling associated with HF. PH associated with HF reduces exercise capacity and carries a poor prognosis, especially when associated with RV dysfunction [1]. The mechanisms responsible for the pulmonary manifestations of chronic HF involve both pulmonary vascular and alveolar septa structural remodeling, characterised by thickening of the capillary endothelial and alveolar epithelial cell basement membranes with abundant proliferation of myofibroblasts (MFs) and excess collagen with reticulin deposition [2-5]. This causes a restrictive lung syndrome contributing to the functional limitation of HF.
Substantial evidence demonstrates that activation of the endothelin (ET) system in HF correlates with disease severity and prognosis [6]. ET-1 is a powerful vasoconstrictor and proliferative peptide produced by endothelin cells and also can be produced from cardiomyocytes in pathological situations. Plasma concentration of ET-1, which may originate from the lungs, is increased in patients with HF $[7,8]$. Indeed, previous studies have demonstrated an upregulation of ET-1 gene expression and increased production of ET-1 in HF lungs [9-11]. The expression of endothelin converting enzyme activity, which is responsible for the conversion of the precursor of ET-1 (big ET-1) into mature ET-1, is also increased in HF lungs [9]. Since the lung is the major site for both clearance and production of ET-1 it may, therefore, represent a preferred target organ for this peptide in HF $[12,13]$. It is currently accepted that lung MFs play a central role in lung fibrotic disorders [14] and our previous results have

\section{AFFILIATIONS}

Research Center, Montreal Heart Institute and Dept of Medicine, Université de Montréal, Montreal, Quebec, Canada.

\section{CORRESPONDENCE}

J. Dupuis

Research Center, Montreal Heart Institute and Dept of Medicine Université de Montréal 5000 Belanger Street Montreal

Quebec H1T 1C8

Canada

E-mail: jocelyn.dupuis@bellnet.ca

Received:

April 072010

Accepted after revision:

June 162010

First published online:

July 012010 
revealed important lung MF proliferation in rats with ischaemic HF [3]. Lung MFs abundantly express ETA and ETB receptors which both stimulate cell proliferation [15]. Other studies also reported that ET-1 can stimulate MFs proliferation, chemotaxis and collagen synthesis [16-18]. Activation of the ET-1 system in HF could, therefore, significantly contribute to lung remodeling and the pulmonary manifestation of left heart disease.

ERAs have demonstrated anti-proliferative and anti-fibroblastic effects $[15,19]$ and their effectiveness for the therapy of certain forms of chronic PAH (idiopathic, familial or associated with connective tissue disease) are already firmly established. Although clinical trials using ERAs in HF such as ENABLE (Endothelin Antagonist Bosentan for Lowering Cardiac Events in Heart Failure) and REACH (Research on Endothelin Antagonism in Chronic Heart Failure) suggested no overall benefit in the treatment of subjects with HF [20], none of these trials specifically evaluated the effect of ERAs on pulmonary structural remodeling, lung function, $\mathrm{PH}$ and RV dysfunction associated with HF. Whether ERAs would be of benefit in the therapy of patients with $\mathrm{HF}$ and significant associated $\mathrm{PH}$ therefore remains unanswered.

In this study, we evaluated the effects of bosentan, a dual ERA, on $\mathrm{PH}$, lung structural remodeling and function and RV function in rats with ischaemic HF.

\section{METHODS}

The study protocol was approved by the animal ethics and research committee of the Montreal Heart Institute (Montreal, Quebec, Canada) and conducted according to guidelines from the Canadian council for the care of laboratory animals. The investigation conforms with the Guide for the Care and Use of Laboratory Animals [21].

\section{Experimental protocol}

Rats were submitted to myocardial infarction (MI) or sham surgery as described previously [22]. Briefly, a lateral thoracotomy was performed and MI was induced by ligating the proximal left anterior descending coronary artery. The sham group was also submitted to thoracotomy but without ligation of the coronary artery. $24 \mathrm{~h}$ after MI surgery the rats were anesthetised with xylazine $\left(10 \mathrm{mg} \cdot \mathrm{kg}^{-1}\right)$ and ketamine $\left(50 \mathrm{mg} \cdot \mathrm{kg}^{-1}\right)$. Blood samples were collected by subclavian vein puncture and immediately centrifuged. Plasma was stored at $-80^{\circ} \mathrm{C}$ until troponin-T concentration was analysed by standard electrochemiluminescence immunoassay using the Cobas e 601 (Roche, Basel, Switzerland). To maximise the likelihood for the development of HF with PH, only the animals with medium to large MI, evaluated by echocardiography 2 weeks after MI surgery, were included in this study. This was defined as left ventricular (LV) wall motion abnormalities involving $>30 \%$ of the segments evaluated in the basal and mid-short axis views. After echocardiographic evaluation, the rats with medium to large MI were randomly divided into three groups: MI+ bosentan treatment group $\left(100 \mathrm{mg} \cdot \mathrm{kg}^{-1} \cdot \mathrm{day}^{-1}\right.$ in food; $\left.\mathrm{n}=13\right)$; MI+bosentan treatment group $\left(200 \mathrm{mg} \cdot \mathrm{kg}^{-1} \cdot \mathrm{day}^{-1}\right.$ in food; $n=11)$; and MI group without treatment $(n=12)$. These therapies lasted for 3 weeks. The sham group received no treatment for the same period $(n=13)$. All animals were maintained on a light-dark $(12 \mathrm{~h} / 12 \mathrm{~h})$ cycle receiving water and food ad libitum.

\section{Transthoracic echocardiographic study}

$\mathrm{LV}$ and RV geometries and functions for all rats were performed 2 and 5 weeks after MI surgery using a phasedarray probe $10 \mathrm{~S}(4.5-11.5 \mathrm{MHz})$ linked to a Vivid 7 system (GE Healthcare Ultrasound, Horten, Norway) [23, 24].

\section{In vivo lung function test and haemodynamic measurements}

After anaesthesia with xylazine $\left(10 \mathrm{mg} \cdot \mathrm{kg}^{-1}\right)$ and ketamine $\left(50 \mathrm{mg} \cdot \mathrm{kg}^{-1}\right)$, the trachea was isolated and connected to a computer-controlled, small-animal ventilator (FlexiVent; Scireq, Montreal, QC, Canada) to evaluate lung function. Lung compliance and elastance were determined and a lung pressurevolume $(\mathrm{P}-\mathrm{V})$ loop was performed and was analysed using the Salazar-Knowles equation:

$$
\mathrm{V}=\mathrm{A}-\mathrm{B} \times \mathrm{e}^{-\mathrm{KP}}
$$

Where $\mathrm{A}$ is the estimate of the inspiratory capacity, B equals total lung capacity minus $\mathrm{V}(P=0)$ and $\mathrm{K}$ is the curvature parameter.

Following this, high-fidelity pressure catheters (Millar Instruments, Houston, TX, USA) were inserted and advanced into the RV and LV to measure the haemodynamics using a powerlab polygraph System (AD Instrument, Colorado Springs, CO, USA).

\section{Morphometric and histological measurements}

The presence of pulmonary oedema was evaluated by measuring the ratio of lung dry/wet weights of the right middle lung lobe. The left lung was perfusion fixed with optimal cutting temperature compound (Sakura, Torrance, CA, USA) and frozen in 2-methylbutane pre-chilled with liquid nitrogen. To quantify collagen deposition in the lungs tissue $(n=7, n=10$, $\mathrm{n}=8$ and $\mathrm{n}=7$ in the sham, MI, MI+Bos100 and MI+Bos200 groups, respectively), Masson's trichrome staining was performed with standard protocols and was analysed using the Image-pro Plus 6.2 software (Media Cybernetics, Bethesda, MD, USA). The proportion of collagen deposition was calculated as the sum of stained collagen tissue divided by the sum of all stained muscle and connective tissue in the visual field.

The heart was removed and dissected. The left and right ventricles were separated and RV hypertrophy (RVH) was assessed by the ratio of the RV/LV+septum weights. For MI, MI+Bos100 and MI+Bos200 rats, the LV scars were dissected and weighed and their surface areas were determined by planimetry.

\section{Statistical analysis}

All values are expressed as mean \pm SEM. The four experimental groups were compared by ANOVA followed by the Fisher's post hoc test for multiple comparisons. Values of $\mathrm{p}<0.05$ were considered to be statistically significant.

\section{RESULTS}

There was no mortality during the 3 weeks treatment period starting 2 weeks after MI in any study group. Baseline echocardiographic LV wall motion abnormality and wall motion score index were comparable among the MI and MI+Bos100 
and MI+Bos200 groups (table 1) and remained similar 5 weeks after MI (table 2). Compared with the sham rats, the $24 \mathrm{~h}$ troponin- $\mathrm{T}$ values were significantly and similarly increased in the MI and MI+Bos100 groups (table 1). In the MI+Bos200 groups, troponin-T was not different compared to the MI+Bos100 group but was slightly and significantly lower than in the MI control group.

\section{Effects of bosentan on systemic haemodynamics, LV remodeling and function}

Heart rates were comparable among all groups. There was decreased mean arterial pressure 5 weeks after MI that was not significantly affected by therapy. Compared with sham $(12 \pm 0.9 \mathrm{mmHg}), \mathrm{HF}$ increased in LV end-diastolic pressure (LVEDP, $28 \pm 3 \mathrm{mmHg})(\mathrm{p}<0.01)$ and nonsignificantly reduced cardiac output as measured by echocardiograph (table 2). Bosentan did not reduce LVEDP at both low and high dosages and did not affect cardiac output. Indices of LV contractility $((+) \mathrm{dP} / \mathrm{dt})$ and relaxation $((-) \mathrm{dP} / \mathrm{dt})$ were reduced after $\mathrm{MI}$ but were not improved by bosentan. Infarct expansion measured from the ratio of scar weight and surface was also unaffected by bosentan therapy (table 3 ).

LV echocardiographic parameters (tables 1 and 2), including LV end-diastolic and end-systolic dimensions, LV end-diastolic and end-systolic areas were significantly increased in the MI group ( $<<0.0001) 2$ and 5 weeks after MI. This was associated with depressed systolic function with significantly reduced LV fractional shorting and LV fractional area change $(p<0.0001)$ in the MI group compared with the sham group. These parameters of LV remodeling and dysfunction were not significantly modified by bosentan treatment at both low and high doses (fig. 1a and b, table 2).

\section{Effects of bosentan on pulmonary haemodynamics, RV remodeling and function}

Compared with sham rats $(23 \pm 0.8 \mathrm{mmHg})$, HF rats developed moderate $\mathrm{PH}$ with increased $\mathrm{RV}$ systolic pressure of $39 \pm 5 \mathrm{mmHg} \quad(\mathrm{p}<0.01)$, while $\mathrm{PH}$ was not improved by bosentan (fig. 2a). Congestive heart failure induced RVH with $\mathrm{RV} / \mathrm{LV}+$ septum weights ratio of $52 \pm 7 \%$ compared to $24 \pm 0.5 \%$ in the sham group $(\mathrm{p}<0.01)$. Bosentan did not change this ratio after therapy (fig. $2 b$ ).

RV echocardiography (tables 1 and 2) demonstrated increased $\mathrm{RV}$ tricuspid valve closing to opening time (TV $\mathrm{c}-\mathrm{o})$ in the MI group compared with the sham group, which were not improved by bosentan treatment. Moreover, RV myocardial performance index (RVMPI) derived from the TVc-o and RV ejection time parameters was greatly increased (worse function) in HF $(\mathrm{p}<0.01)$ and was not improved by bosentan therapy (fig. 1c). Furthermore, RV systolic function measured by tricuspid annulus plane systolic excursion (TAPSE) was significantly reduced in the MI group and was not improved by bosentan (fig. 1d).

\section{Effects of bosentan on pulmonary structural remodeling and function}

The ratio of the wet lung weight/body weight increased by $\sim 66 \%$ after $\mathrm{MI}(\mathrm{p}<0.01)$ (fig. $3 \mathrm{a})$ and the dry lung/body weight ratio was increased by $55 \% \quad(p<0.01)$ providing evidence of substantial pulmonary remodeling: treatment with bosentan did not reverse the increased ratio (fig. 3b). The dry/ wet lung weight ratio was, however, comparable among all groups, suggesting that no significant pulmonary oedema occurred (fig. 3c). The lung tissue collagen deposition (fig. 4) was greater in MI than sham rats $(14.5 \pm 3.0 \%$ versus $5.7 \pm 0.5 \%$;

TABLE 1 Baseline of $24 \mathrm{~h}$ troponin-T and 2 weeks echocardiographic parameters

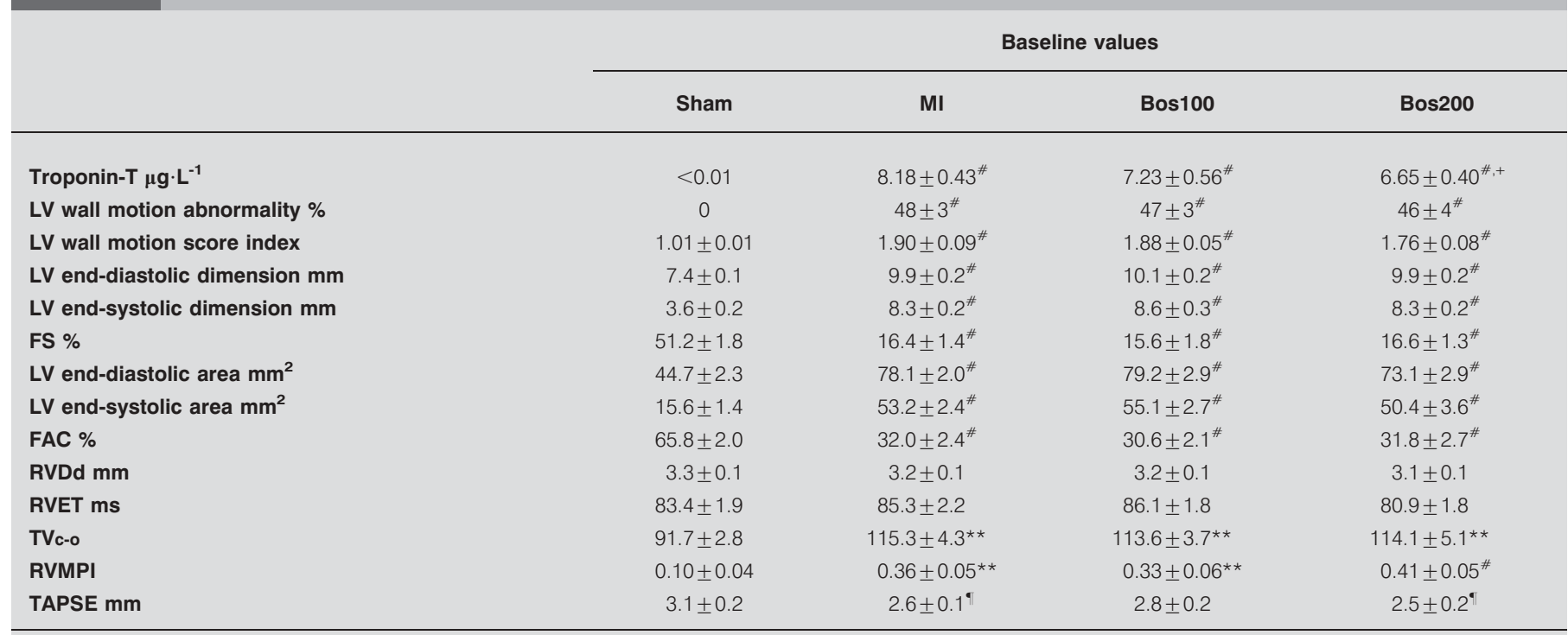

Data are presented as mean \pm SEM. MI: myocardial infarction; Bos100: bosentan $100 \mathrm{mg} \cdot \mathrm{kg}^{-1} \cdot \mathrm{day}^{-1}$; Bos200: bosentan $200 \mathrm{mg} \cdot \mathrm{kg}^{-1} \cdot \mathrm{day}^{-1}$; $\mathrm{LV}$ : left ventricular; FS: fractional shortening; FAC: Fractional area changes; RVDd: right ventricular end-diastolic dimension; RVET: right ventricular ejection time; TVc-o: tricuspid valve closing to opening; RVMPI: right ventricular myocardial performance index; TAPSE: tricuspid annulus plane systolic excursion. ${ }^{*}: p<0.0001$ versus sham; ${ }^{*}: p<0.05$ versus sham; ${ }^{+}: p<0.05$ versus Ml. **: $p<0.01$ versus sham. 
TABLE 2 Effect of bosentan on 5 weeks echocardiographic parameters

\begin{tabular}{|c|c|c|c|c|}
\hline Cardiac output $\mathrm{mL} \cdot \mathrm{min}^{-1}$ & $140 \pm 5$ & $120 \pm 11$ & $119 \pm 13$ & $114 \pm 7$ \\
\hline LV wall motion score index & $1.00 \pm 0$ & $1.96 \pm 0.05^{\#}$ & $2.00 \pm 0.07^{\#}$ & $1.87 \pm 0.07^{\#}$ \\
\hline LV end-diastolic dimension $\mathrm{mm}$ & $7.6 \pm 0.2$ & $10.9 \pm 0.2^{\#}$ & $10.9 \pm 0.2^{\#}$ & $11.2 \pm 0.2^{\#}$ \\
\hline LV end-systolic dimension mm & $3.7 \pm 0.2$ & $9.3 \pm 0.2^{\#}$ & $9.3 \pm 0.3^{\#}$ & $9.5 \pm 0.3^{\#}$ \\
\hline RVDd mm & $3.5 \pm 0.1$ & $3.5 \pm 0.1$ & $3.6 \pm 0.2$ & $3.6 \pm 0.1$ \\
\hline RVET ms & $83.4 \pm 2.5$ & $83.6 \pm 2.9$ & $86.4 \pm 1.8$ & $80.7 \pm 2.3$ \\
\hline TVc-o & $97.1 \pm 4.1$ & $120.1 \pm 5.1^{\text {** }}$ & $127.9 \pm 8.1^{\text {** }}$ & $113.9 \pm 5.4$ \\
\hline
\end{tabular}

Data are presented as mean \pm SEM. MI: myocardial infarction; Bos100: bosentan $100 \mathrm{mg} \cdot \mathrm{kg}^{-1} \cdot \mathrm{day}^{-1}$; Bos200: bosentan $200 \mathrm{mg} \cdot \mathrm{kg}^{-1} \cdot \mathrm{day}^{-1} ; \mathrm{LV}$ : left ventricular; RVDd: right ventricular end-diastolic dimension; RVET: right ventricular ejection time; TVc-o: tricuspid valve closing to opening. ${ }^{* *} p<0.01$ versus sham; ${ }^{*}: p<0.0001$ versus sham

$\mathrm{p}<0.01)$ and unaffected by bosentan treatment $(14.2 \pm 3.3 \%$ at $100 \mathrm{mg}$ and $14.4 \pm 2.9 \%$ at $200 \mathrm{mg}$ ).

Heart failure significantly reduced pulmonary function with a decreased compliance (fig. 5a) and caused a restrictive lung syndrome with a downward shift of the lungs $\mathrm{P}-\mathrm{V}$ loop (fig. 5b); this dysfunction was not improved by bosentan.

\section{DISCUSSION}

We evaluated the effects of endothelin receptor blockade on $\mathrm{PH}$, lung structural remodeling and pulmonary function in rats with ischaemic HF. HF induced secondary $\mathrm{PH}$ with lung dysfunction and important structural remodeling characterised by excessive collagen deposition. This was associated with the development of RV dysfunction and RVH. Therapy with the dual ERA bosentan did not improve $\mathrm{PH}$, lung structural remodeling and lung dysfunction and also did not prevent RVH. Bosentan also had no effect on LV remodeling and function as scar size and echocardiographic parameters of
LV function remained largely unchanged. These data suggest that endothelin receptors do not play central roles on $\mathrm{PH}$ and lung structural remodeling associated with ischaemic HF in rats.

\section{Effects of bosentan on lung structural remodeling and function}

The repair process in response to lung injury is characterised by the proliferation of MFs that can originate from resident lung cells or marrow-derived cells [14, 25, 26]. These cells are important modulators of lung fibrotic disorders and also play an important role in lung structural remodeling associated with HF [3]. MFs abundantly express both ETA and ETB receptors and both receptors stimulate isolated lung MF proliferation in response to ET-1 stimulation [15]. Lungs from HF rats exhibit higher mRNA levels of collagen, fibronectin and transforming growth factor- $\beta 1$ and - $\beta 3$ [21]. KAPANCI et al. [27] previously demonstrated alveolar septa proliferation of MFs in human post-capillary $\mathrm{PH}$, non-observable in pre-capillary

TABLE 3 Effect of bosentan on haemodynamic and morphometric parameters

\begin{tabular}{|c|c|c|c|c|}
\hline $\mathrm{HR}$ beats $\mathrm{min}^{-1}$ & $236 \pm 12$ & $256 \pm 9$ & $238 \pm 12$ & $240 \pm 7$ \\
\hline LVEDP mmHg & $12 \pm 0.9$ & $28 \pm 3^{* *}$ & $22 \pm 4^{*}$ & $22 \pm 3^{*}$ \\
\hline LV $(+) \mathrm{dP} / \mathrm{dt} \mathrm{mmHg} \cdot \mathrm{s}^{-1}$ & $6924 \pm 490$ & $4913 \pm 552^{\star \star}$ & $5551 \pm 494^{*}$ & $4956 \pm 163^{* *}$ \\
\hline LV (-)dP/dt mmHg $\cdot \mathrm{s}^{-1}$ & $5264 \pm 402$ & $2896 \pm 313^{\#}$ & $3218 \pm 310^{\#}$ & $3175 \pm 143^{\#}$ \\
\hline Body weight g & $429 \pm 10$ & $424 \pm 14$ & $422 \pm 16$ & $424 \pm 7$ \\
\hline Scar weight $\mathbf{g}$ & NA & $0.12 \pm 0.01^{\#}$ & $0.13 \pm 0.01^{\#}$ & $0.11 \pm 0.01^{\#}$ \\
\hline Scar/body weight $\%$ & NA & $0.03 \pm 0.00^{\#}$ & $0.03 \pm 0.00^{\#}$ & $0.03 \pm 0.00^{\#}$ \\
\hline Scar surface $\mathrm{mm}^{2}$ & NA & $111.7 \pm 3.7^{\#}$ & $120.8 \pm 9.0^{\#}$ & $108.7 \pm 5.7^{\#}$ \\
\hline Scar weight/scar surface $\mathbf{g} \cdot \mathrm{mm}^{-2}$ & NA & $0.09 \pm 0.01^{\#}$ & $0.09 \pm 0.01^{\#}$ & $0.11 \pm 0.00^{\#}$ \\
\hline
\end{tabular}

Data are presented as mean \pm SEM. MI: myocardial infarction; Bos100: bosentan $100 \mathrm{mg} \cdot \mathrm{kg}^{-1} \cdot \mathrm{day}^{-1}$; Bos200: bosentan $200 \mathrm{mg} \cdot \mathrm{kg}^{-1} \cdot \mathrm{day}^{-1}$; HR: heart rate; MAP: mean arterial pressure; LVEDP: left ventricular (LV) end diastolic pressure; RV: right ventricular; NA: not applicable. ${ }^{*}: p<0.05$ versus sham; ${ }^{* *}$ : $p<0.01$ versus sham; \#: $p<0.0001$ versus sham. 

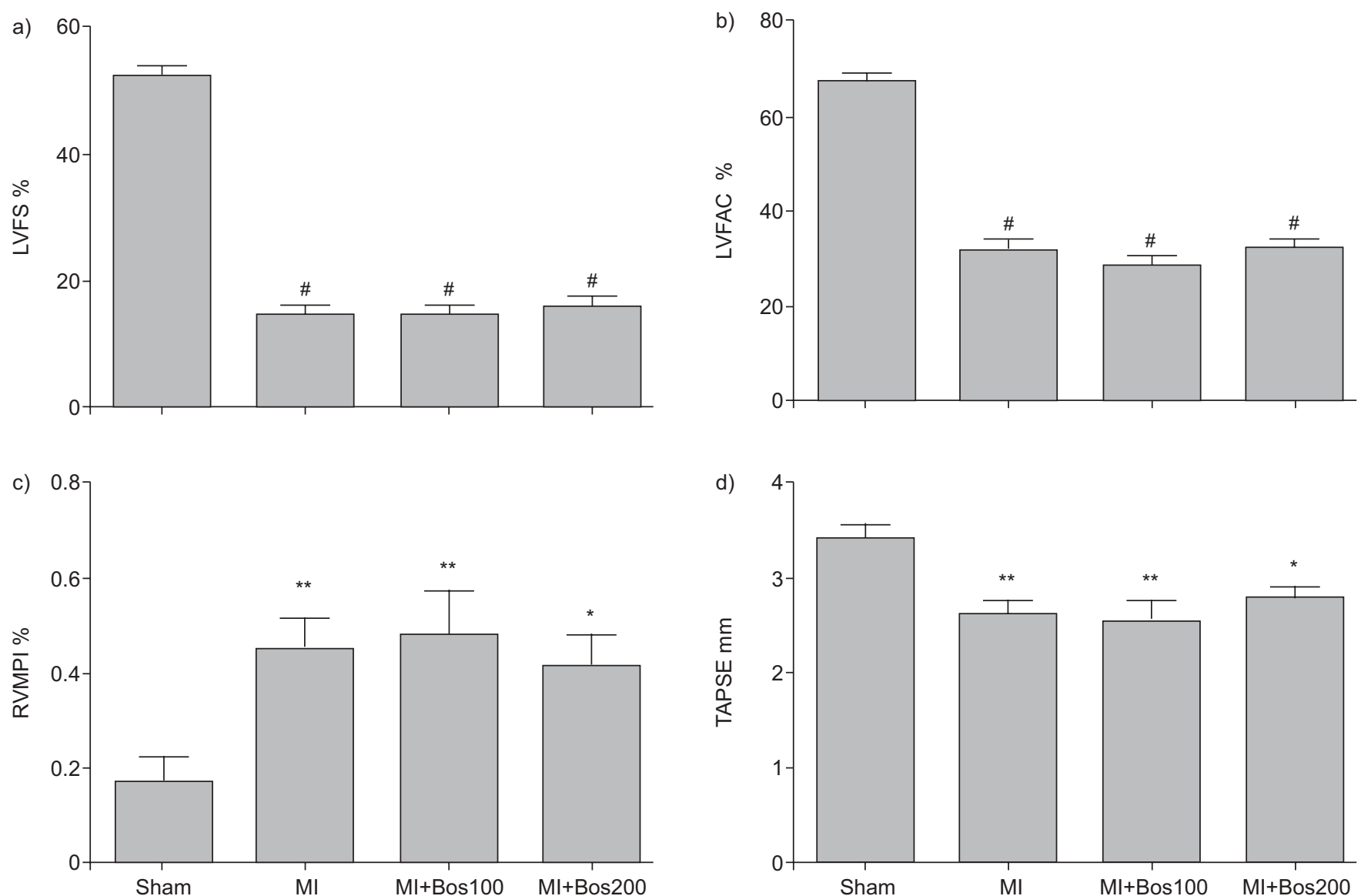

FIGURE 1. Effect of bosentan on a) left ventricular fractional shorting (LVFS), b) left ventricular fractional area changing (LVFAC), c) right ventricular myocardial performance index (RVMPI) and d) tricuspid annulus plane systolic excursion (TAPSE) after treatment in sham, myocardial infarction (MI), Ml+Bos100 (bosentan $100 \mathrm{mg} \cdot \mathrm{kg}^{-1} \cdot \mathrm{day}^{-1}$ ) and Ml+Bos200 (bosentan $200 \mathrm{mg} \cdot \mathrm{kg}^{-1} \cdot$ day $^{-1}$ ) rats. Results are expressed as mean \pm SEM. *: $\mathrm{p}<0.05$ versus sham; **: $p<0.01$ versus sham; ${ }^{*}: \mathrm{p}<0.0001$ versus sham

PH. Although MF proliferation and fibrosis could play an initially protective role against the deleterious increase in capillary pressure and prevent the development of alveolar oedema, in the longer term, however, this response probably becomes maladaptive and contributes to a restrictive lung syndrome, $\mathrm{PH}$ and $\mathrm{RVH}$.
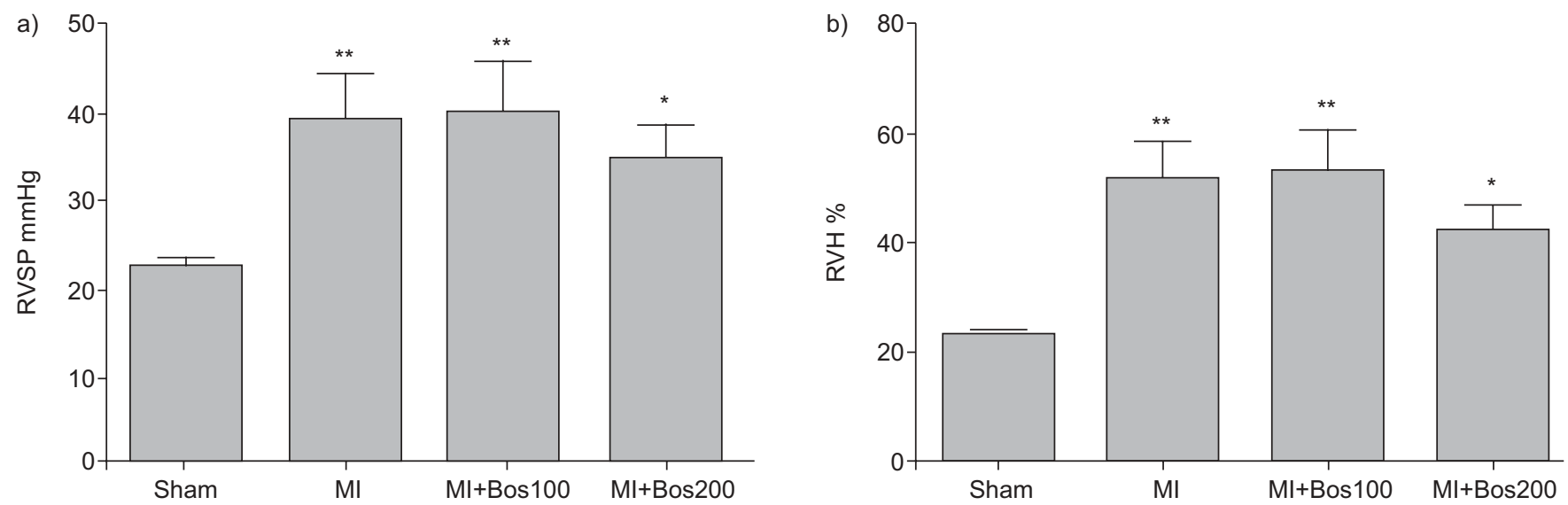

FIGURE 2. Effect of bosentan on a) right ventricular systolic pressure (RVSP) and b) right ventricular hypertrophy (RVH) after treatment in sham, myocardial infarction (MI), Ml+Bos100 (bosentan $100 \mathrm{mg} \cdot \mathrm{kg}^{-1} \cdot \mathrm{day}^{-1}$ ) and Ml+Bos200 (bosentan $200 \mathrm{mg} \cdot \mathrm{kg}^{-1} \cdot \mathrm{day}^{-1}$ ) rats. Results are expressed as mean $\pm \mathrm{SEM}$. ${ }^{*}: \mathrm{p}<0.05$ versus sham; ${ }^{* *}$ : $\mathrm{p}<0.01$ versus sham. 

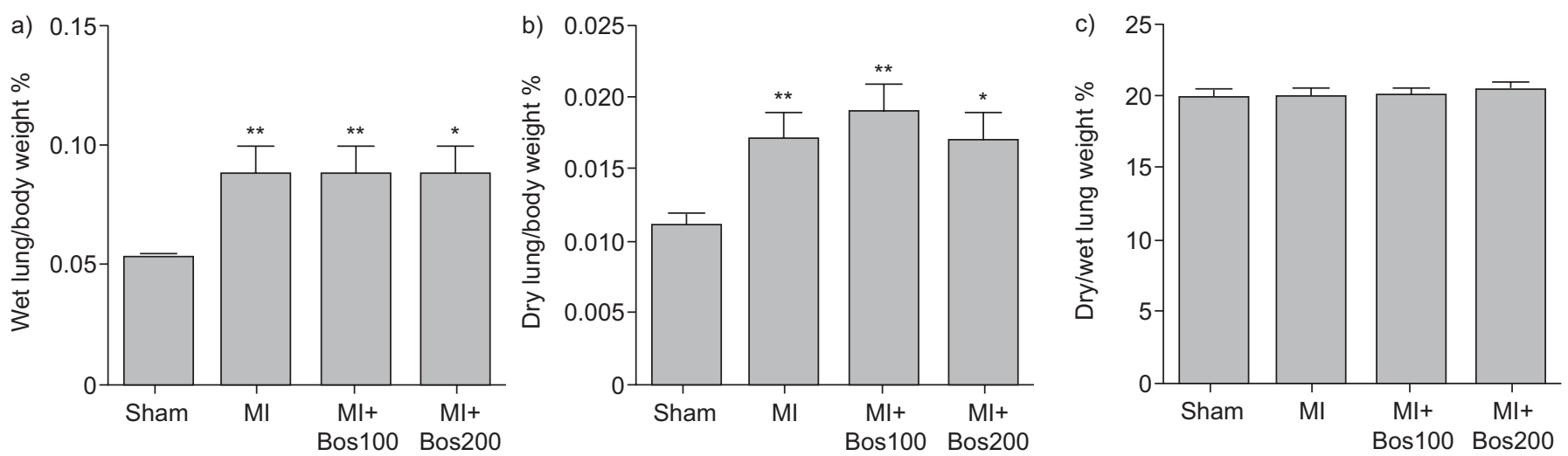

FIGURE 3. Effect of bosentan on a) wet lung weight/body weight, b) dry lung weight/body weight and c) dry/wet lung weight ratios after treatment in sham, myocardial infarction (MI), Ml+Bos100 (bosentan $100 \mathrm{mg} \cdot \mathrm{kg}^{-1} \cdot$ day $^{-1}$ ) and Ml+Bos200 (bosentan $200 \mathrm{mg} \cdot \mathrm{kg}^{-1} \cdot$ day $^{-1}$ ) rats. Results are expressed as mean \pm SEM. *: $\mathrm{p}<0.05$ versus sham; $* *: p<0.01$ versus sham.

We found that HF induced significant pulmonary remodeling with increased lung weight and excessive lung collagen deposition but this was not improved by the treatment with bosentan. These results suggest that dual ERA therapy with bosentan might not be useful for the treatment of $\mathrm{PH}$ and lung remodeling associated with HF. Although ERAs have been demonstrated to have anti-proliferative and anti-fibroblastic effects [15, 19], other more important and predominant mechanism may be involved in the lung remodeling of HF. Furthermore, since the therapy was started 2 weeks after MI when lung structural remodeling was already well established, therapy may have been unable to reverse the disease process at this more advanced stage.

\section{Effects of bosentan on $P H$, and $R V$ and $L V$ remodeling and function}

MF proliferation and excessive collagen deposition in the lung contribute to the development of secondary $\mathrm{PH}$ and $\mathrm{RVH}$ $[3,26]$. Although bosentan has demonstrated its capacity to improve chronic PAH of various aetiologies, its effectiveness in $\mathrm{PH}$ associated with left heart disease has not been specifically tested. Herein, we demonstrated that bosentan had no detectable
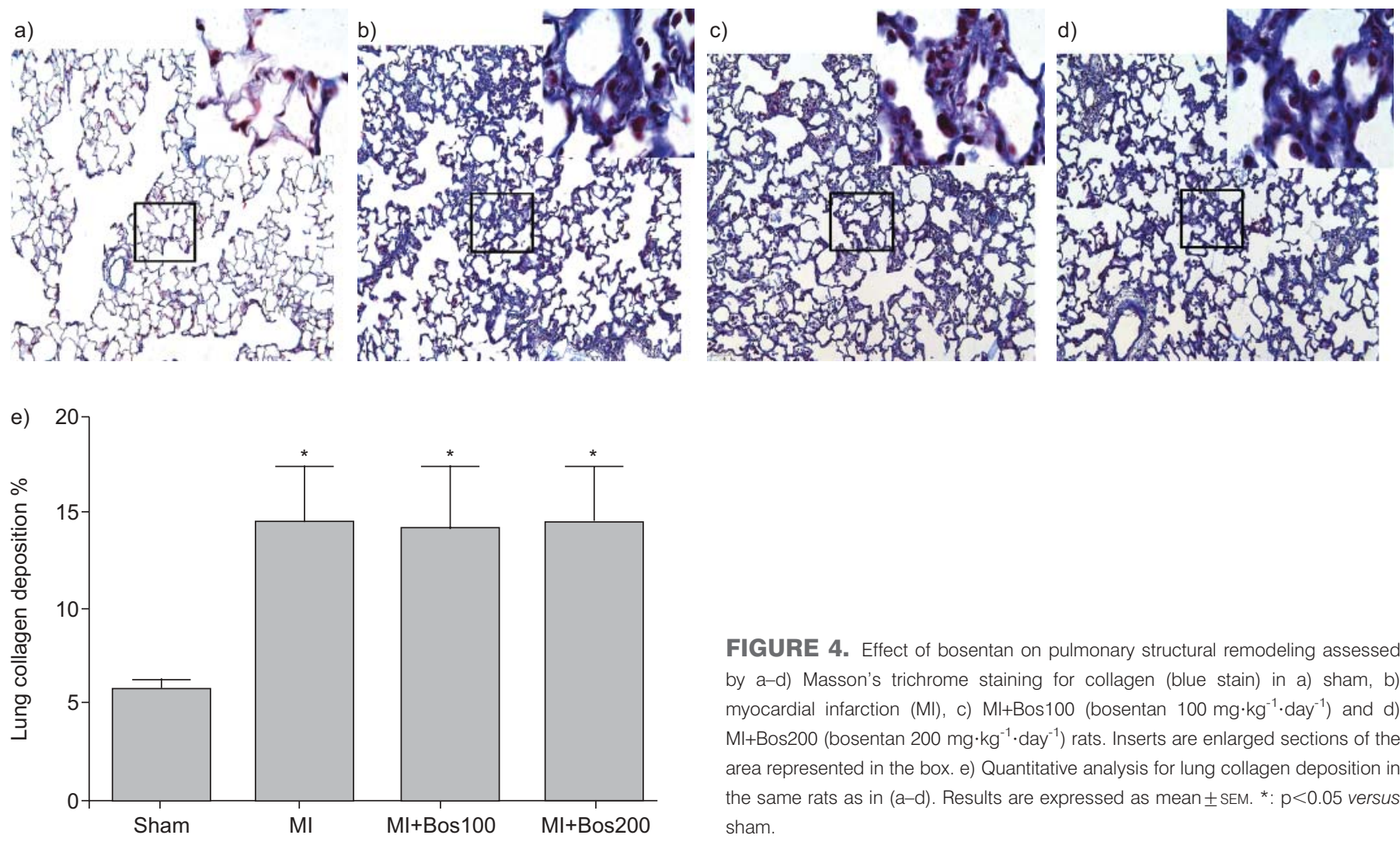

FIGURE 4. Effect of bosentan on pulmonary structural remodeling assessed by a-d) Masson's trichrome staining for collagen (blue stain) in a) sham, b) myocardial infarction (MI), c) Ml+Bos100 (bosentan $100 \mathrm{mg} \cdot \mathrm{kg}^{-1} \cdot \mathrm{day}^{-1}$ ) and d) Ml+Bos200 (bosentan $200 \mathrm{mg} \cdot \mathrm{kg}^{-1} \cdot$ day $^{-1}$ ) rats. Inserts are enlarged sections of the area represented in the box. e) Quantitative analysis for lung collagen deposition in the same rats as in $(a-d)$. Results are expressed as mean \pm SEM. ${ }^{*}: p<0.05$ versus sham. 

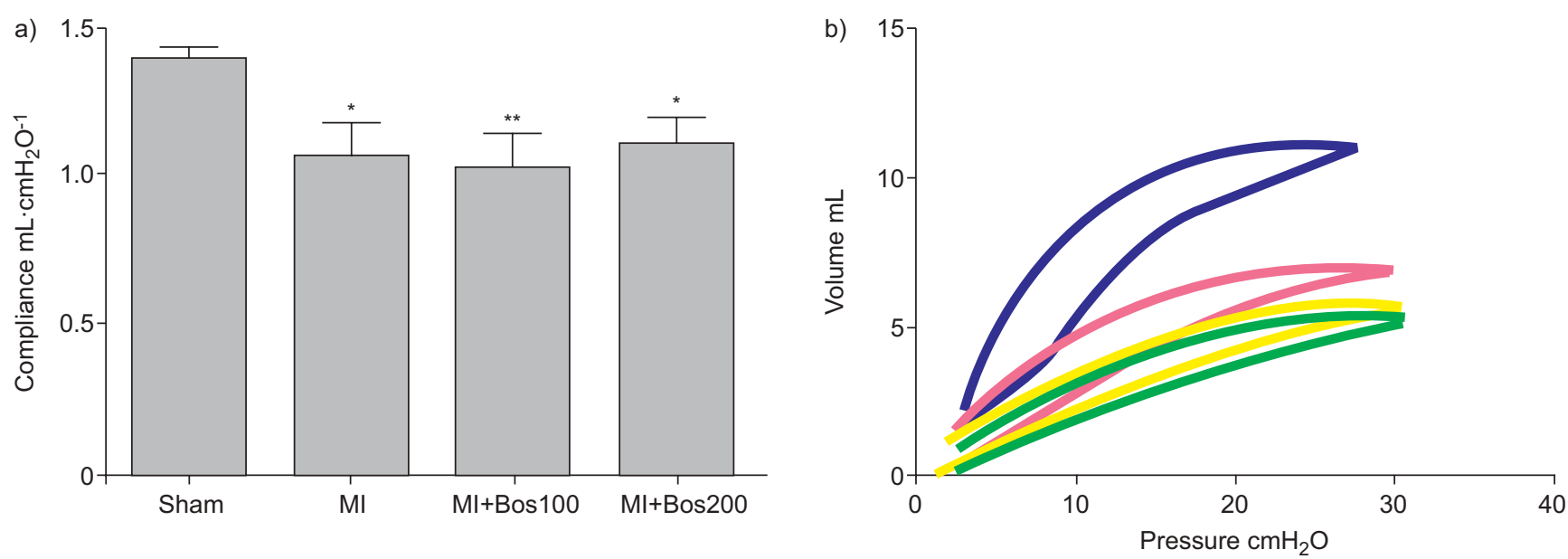

FIGURE 5. Effect of bosentan on lung function a) on compliance and b) by pressure-volume loop technique after treatment in sham, myocardial infarction (MI), Ml+Bos100 (bosentan $100 \mathrm{mg} \cdot \mathrm{kg}^{-1} \cdot \mathrm{day}^{-1}$ ) and Ml+Bos200 (bosentan $200 \mathrm{mg} \cdot \mathrm{kg}^{-1} \cdot$ day $^{-1}$ ) rats. Results are expressed as mean \pm SEM. Blue line: sham; pink line: Ml; yellow line Bos100; green line: Bos200. *: $p<0.05$ versus sham; **: $p<0.01$ versus sham.

effect on PH associated with LV systolic dysfunction. Furthermore, RV function as measured by both RVMPI and TAPSE, as well as RVH measured by $\mathrm{RV} / \mathrm{LV}+$ septum weight were not improved. A previous study [22] has shown that administration of the selective ETA receptor antagonist LU-135252 (darusentan) $24 \mathrm{~h}$ after coronary artery ligation in the rat MI model significantly reduced the severity of $\mathrm{PH}$, but failed to reduce scar size, lung fibrosis and RVH and did not prevent medial hypertrophy of resistance pulmonary arteries $(50 \mu \mathrm{M}$ to $200 \mu \mathrm{M})$. This would suggest that ET receptor selectivity does not influence the effect of ERA therapy on lung remodeling in HF.

In our study therapy was started 2 weeks after MI, when LV scarring was already well established. Although $24 \mathrm{~h}$ troponin-T values were slightly lower in the Bos200 group, 2-week baseline infarct size and LV function measured by cardiac ultrasound were measured prior to initiation of therapy and were similar. Bosentan had no effect of on LVEDP or LV $( \pm) \mathrm{dP} / \mathrm{dt}$ and also did not improve the LV function and geometry as evaluated by echocardiography after 5 weeks. This is consistent with previous studies in the same model [28] demonstrating the absence of improvement of left ventricular dysfunction after treatment with non-selective ERAs.

\section{Limitations and basic and clinical relevance of this study}

Use of the rat MI model in the development of drugs for the therapy of ischaemic HF has been validated. Despite conflicting results in pre-clinical studies, ERAs were initially developed for the therapy of HF and are still being contemplated as potentially effective therapies. The randomised trials ENABLE and REACH suggested no overall benefit of ERA therapy with bosentan in the treatment of patients with HF [20]. It was suggested that some subjects had initial deterioration due to fluid retention, but later improved. The EARTH (Endothelin A Receptor Antagonist Trial in Heart Failure) trial using the selective ETA antagonist darusentan [29] also showed no effect on cardiac remodeling or clinical symptoms. However, none of these trials were specifically designed to address the potential role of ERAs on PH and lung structural remodeling, thus, it remained possible that a subset of subjects with associated $\mathrm{PH}$ may derive benefit from ERA therapy. $\mathrm{PH}$ is a frequent complication of HF that carries a poor prognosis. In a prospective study of 377 consecutive class II to IV patients referred to a HF clinic, $\mathrm{PH}$ was found at catheterisation in 236 patients (62\%, mean pulmonary arterial pressure PAP $>20 \mathrm{mmHg})$ [1] and, together with its impact on RV function, was independently associated with reduced survival. In another similar study, more than moderate $\mathrm{PH}$ ( $>2.5$ Wood units) was found in $36 \%$ of subjects and associated with reduced peak oxygen uptake during exercise [30]. Death and hospitalisation for HF were increased in subjects with echocardiographic evidence of PH [31]. Previous analysis of the ENABLE trial has raised the possibility that if only HF patients with increased pulmonary arterial pressures had been included, a clear benefit of treatment with ET antagonists may have emerged [32]. The current pre-clinical study carefully selected rats that developed moderate $\mathrm{PH}$ and found no benefit of bosentan on $\mathrm{PH}, \mathrm{RVH}$, lung remodeling and fibrosis, as well as on RV function and respiratory function.

Therefore, our results argue against a significant role of ERAs for the therapy of both LV failure and its associated $\mathrm{PH}$ despite the demonstrated activation of the ET system, with increased plasma ET levels correlating with the severity of HF and associated $\mathrm{PH}$. Our results do not contradict the possibility that the activation of the endothelin system may be deleterious and contribute to the pathophysiology of PH associated with HF. The demonstration that pharmacological blockade of the ET receptors has no effect could suggest a minor role, or that alternate pathways play a more dominant role, such as the renin-angiotensin system for example.

There is indeed basic and clinical evidence that other therapies could beneficially alter lung remodeling in HF. We recently demonstrated in the same animal model that the HMG-CoA reductase inhibitor atorvastatin importantly reduced lung remodeling and fibrosis and improved respiratory and RV function in this model [33]. Similarly, angiotensin receptor blockade with irbesartan started early after MI also prevented lung remodeling [3]. This is consistent with clinical studies 
demonstrating the benefit of angiotensin-converting enzyme inhibition on lung function of subjects with HF [34]. Unfortunately, other previous pre-clinical studies evaluating currently approved therapies for HF did not specifically and extensively measure RV haemodynamics and functions in addition to lung morphology and functions. Nevertheless, some indices suggest that these therapies could potentially have beneficial effects on lung and RV functions. A study comparing the beta-blockers carvedilol and metoprolol used in equal $\beta$-adrenoreceptor blocking potency found that both improved LV haemodynamics, but that only carvedilol reduced myocardial fibrosis and RV hypertrophy [35]: an effect possibly attributed to the $\alpha$-adrenoreceptor blocking properties and anti-oxidant effects of carvedilol. In another study using the same model, aldosterone antagonism with spironolactone administered early after coronary ligation improved LV remodeling and, interestingly, almost normalised the important increase in lung weight [36]. RV haemodynamics and lung function were, however, not evaluated and these findings were not discussed by the authors. Whether or not aldosterone antagonism could selectively target the lung in HF remains speculative, but a recent study randomised 30 subjects with $\mathrm{HF}$ to spironolactone or placebo for 6 months and found that active treatment improved exercise capacity and increased diffusing capacity of the lung for carbon monoxide due to an improvement in alveolar-capillary membrane diffusing capacity [37]. Collectively, these data suggest that some therapies used in HF could have direct or indirect effects on lung remodeling, $\mathrm{PH}$ and $\mathrm{RV}$ function.

The smaller dosage of bosentan used in this trial $\left(100 \mathrm{mg} \cdot \mathrm{kg}^{-1}\right.$. day $^{-1}$ ) has previously been shown to be effective in numerous pathological lung models including $\mathrm{PAH}$ induced hypoxia [38], bleomycin lung fibrosis [39] and emphysema [40]. Since we also used a higher dosage $\left(200 \mathrm{mg} \cdot \mathrm{kg}^{-1} \cdot\right.$ day $\left.^{-1}\right)$, it is unlikely that insufficient dosing was responsible for the lack of benefit in our study. Finally, bosentan is a dual ET receptor antagonist and we can not exclude that other ERAs with different ETA/ETB receptor selectivity could provide some benefit in $\mathrm{PH}$ associated with HF. However, in PAH, both ETA and ETB antagonists have demonstrated benefits but whether ET receptor selectivity translates into any clinically significant advantage has not been demonstrated and is still a subject of debate.

\section{Conclusions}

Rats with ischaemic heart failure developed moderate PH with important lung structural remodeling characterised by excessive collagen deposition and associated with RV dysfunction and RVH. These changes are not reversed by ERA therapy using bosentan. These findings suggest that endothelin receptors are unlikely to provide benefit for the treatment of $\mathrm{PH}$, lung remodeling and RV dysfunction associated with HF.

\section{SUPPORT STATEMENT}

This study was supported by the Canadian Institutes of Health Research and the Montreal Heart Institute Foundation. J. Dupuis is a National Researcher of the "Fonds de la Recherche en Santé du Québec". J-C. Tardif holds the Canadian Pfizer Research Chair on atherosclerosis.

\section{STATEMENT OF INTEREST}

A statement of interest for J. Dupuis can be found at www.erj. ersjournals.com/site/misc/statements.xhtml

\section{ACKNOWLEDGEMENTS}

We would like to thank E. Dedelis, D. Belzile and Q.T. Nguyen (all Research Center of the Montreal Heart Institute, Montreal, Quebec, Canada) for their expert technical assistance.

\section{REFERENCES}

1 Ghio S, Gavazzi A, Campana C, et al. Independent and additive prognostic value of right ventricular systolic function and pulmonary artery pressure in patients with chronic heart failure. J Am Coll Cardiol 2001; 37: 183-188.

2 Huang W, Kingsbury MP, Turner MA, et al. Capillary filtration is reduced in lungs adapted to chronic heart failure: morphological and haemodynamic correlates. Cardiovasc Res 2001; 49: 207-217.

3 Jasmin JF, Calderone A, Leung TK, et al. Lung structural remodeling and pulmonary hypertension after myocardial infarction: complete reversal with irbesartan. Cardiovasc Res 2003; 58: 621-631.

4 Kingsbury MP, Huang W, Donnelly JL, et al. Structural remodelling of lungs in chronic heart failure. Basic Res Cardiol 2003; 98: 295-303.

5 Gehlbach BK, Geppert E. The pulmonary manifestations of left heart failure. Chest 2004; 125: 669-682.

6 Puri S, Baker BL, Dutka DP, et al. Reduced alveolar-capillary membrane diffusing capacity in chronic heart failure. Its pathophysiological relevance and relationship to exercise performance. Circulation 1995; 91: 2769-2774.

7 Wei CM, Lerman A, Rodeheffer RJ, et al. Endothelin in human congestive heart failure. Circulation 1994; 89: 1580-1586.

8 Dupuis J. Increased endothelin levels in congestive heart failure: does it come from the lungs? Does it matter? Cardiovasc Res 2004; 63: 5-7.

9 von Lueder TG, Kjekshus H, Edvardsen T, et al. Mechanisms of elevated plasma endothelin-1 in CHF: congestion increases pulmonary synthesis and secretion of endothelin-1. Cardiovasc Res 2004; 63: 41-50.

10 Lepailleur-Enouf D, Egidy G, Philippe M, et al. Pulmonary endothelinergic system in experimental congestive heart failure Cardiovasc Res 2001; 49: 330-339.

11 Giaid A, Yanagisawa M, Langleben D, et al. Expression of endothelin-1 in the lungs of patients with pulmonary hypertension. N Engl J Med 1993; 328: 1732-1739.

12 Dupuis J, Stewart DJ, Cernacek P, et al. Human pulmonary circulation is an important site for both clearance and production of endothelin-1. Circulation 1996; 94: 1578-1584.

13 Staniloae C, Dupuis J, White M, et al. Reduced pulmonary clearance of endothelin in congestive heart failure: a marker of secondary pulmonary hypertension. J Card Fail 2004; 10: 427-432.

14 Hinz B, Phan SH, Thannickal VJ, et al. The myofibroblast: one function, multiple origins. Am J Pathol 2007; 170: 1807-1816.

15 Prefontaine A, Calderone A, Dupuis J. Role of endothelin receptors on basal and endothelin-1-stimulated lung myofibroblast proliferation. Can J Physiol Pharmacol 2008; 86: 337-342.

16 Gallelli L, Pelaia G, D'Agostino B, et al. Endothelin-1 induces proliferation of human lung fibroblasts and IL-11 secretion through an ET(A) receptor-dependent activation of MAP kinases. J Cell Biochem 2005; 96: 858-868.

17 Shi-Wen X, Denton CP, Dashwood MR, et al. Fibroblast matrix gene expression and connective tissue remodeling: role of endothelin-1. J Invest Dermatol 2001; 116: 417-425.

18 Peacock AJ, Dawes KE, Shock A, et al. Endothelin-1 and endothelin-3 induce chemotaxis and replication of pulmonary artery fibroblasts. Am J Respir Cell Mol Biol 1992; 7: 492-499.

19 Clozel M, Salloukh H. Role of endothelin in fibrosis and antifibrotic potential of bosentan. Ann Med 2005; 37: 2-12. 
20 Packer M, McMurray J, Massie BM, et al. Clinical effects of endothelin receptor antagonism with bosentan in patients with severe chronic heart failure: results of a pilot study. J Card Fail 2005; 11: 12-20.

21 National Research Council. Guide for the Care and Use of Laboratory Animals. NIH Publication No. 85-23. US National Institutes of Health , 1985.

22 Nguyen QT, Colombo F, Rouleau JL, et al. LU135252, an endothelin(A) receptor antagonist did not prevent pulmonary vascular remodelling or lung fibrosis in a rat model of myocardial infarction. Br J Pharmacol 2000; 130: 1525-1530.

23 Litwin SE, Katz SE, Morgan JP, et al. Serial echocardiographic assessment of left ventricular geometry and function after large myocardial infarction in the rat. Circulation 1994; 89: 345-354.

24 Tei C, Dujardin KS, Hodge DO, et al. Doppler echocardiographic index for assessment of global right ventricular function. J Am Soc Echocardiogr 1996; 9: 838-847.

25 Scotton CJ, Chambers RC. Molecular targets in pulmonary fibrosis: the myofibroblast in focus. Chest 2007; 132: 1311-1321.

26 Dupuis J, Préfontaine A, Villeneuve L, et al. Bone marrow-derived progenitor cells contribute to lung remodelling after myocardial infarction. Cardiovascular Pathology 2007; 16: 321-328.

27 Kapanci Y, Burgan S, Pietra GG, et al. Modulation of actin isoform expression in alveolar myofibroblasts (contractile interstitial cells) during pulmonary hypertension. Am J Pathol 1990; 136: 881-889.

28 Nguyen QT, Cernacek P, Sirois MG, et al. Long-term effects of nonselective endothelin A and B receptor antagonism in postinfarction rat: importance of timing. Circulation 2001; 104: 2075-2081.

29 Anand I, McMurray J, Cohn JN, et al. Long-term effects of darusentan on left-ventricular remodelling and clinical outcomes in the Endothelin A Receptor Antagonist Trial in Heart Failure (EARTH): randomised, double-blind, placebo-controlled trial. Lancet 2004; 364: 347-354.
30 Butler J, Chomsky DB, Wilson JR. Pulmonary hypertension and exercise intolerance in patients with heart failure. J Am Coll Cardiol 1999; 34: 1802-1806.

31 Abramson SV, Burke JF, Kelly JJ Jr, et al. Pulmonary hypertension predicts mortality and morbidity in patients with dilated cardiomyopathy. Ann Intern Med 1992; 116: 888-895.

32 Kelland NF, Webb DJ. Clinical trials of endothelin antagonists in heart failure: a question of dose? Exp Biol Med (Maywood) 2006; 231: 696-699.

33 Jiang BH, Tardif J-C, Sauvageau S, et al. Beneficial effects of atorvastatin on lung structural remodeling and function in ischemic heart failure. J Card Fail 2010; 16: 679-688.

34 Guazzi M, Agostoni P. Angiotensin-converting enzyme inhibition restores the diffusing capacity for carbon monoxide in patients with chronic heart failure by improving the molecular diffusion across the alveolar capillary membrane. Clin Sci (Lond) 1999; 96: 17-22.

35 Wei S, Chow LT, Sanderson JE. Effect of carvedilol in comparison with metoprolol on myocardial collagen postinfarction. J Am Coll Cardiol 2000; 36: 276-281.

36 Mulder P, Mellin V, Favre J, et al. Aldosterone synthase inhibition improves cardiovascular function and structure in rats with heart failure: a comparison with spironolactone. Eur Heart J 2008; 29: 2171-2179.

37 Agostoni P, Magini A, Andreini D, et al. Spironolactone improves lung diffusion in chronic heart failure. Eur Heart J 2005; 26: 159-164.

38 Chen SJ, Chen YF, Meng QC, et al. Endothelin-receptor antagonist bosentan prevents and reverses hypoxic pulmonary hypertension in rats. J Appl Physiol 1995; 79: 2122-2131.

39 Schroll S, Arzt M, Sebah D, et al. Improvement of bleomycininduced pulmonary hypertension and pulmonary fibrosis by the endothelin receptor antagonist Bosentan. Respir Physiol Neurobiol 2010; 170: 32-36.

40 Chen Y, Hanaoka M, Droma Y, et al. Endothelin-1 receptor antagonists prevent the development of pulmonary emphysema in rats. Eur Respir J 2010; 35: 904-912. 